\title{
IMMUNITY
}

\section{Antitumor role for Defensin in flies}

Parvy et al. eLife 8, e45061 (2019)

Antimicrobial peptides (AMPs) are an important part of the innate immune system and the first line of defense against a variety of pathogens. Increasing evidence suggests that AMPs are involved in other important physiological functions such as aging or wound healing, but these findings still need to be validated in vivo. In a new study published in eLife, a team led by Julia Cordero from the Wolfson Wohl Cancer Research Centre and CRUK Beatson Institute in Glasgow, UK, shows that the AMP Defensin can induce tumor cell death in Drosophila, thereby providing the first validation of the role of an AMP as an anticancer agent in a living organism.

"Many AMPs had already been shown to induce tumor cell death mostly in cancer cell cultures making them interesting targets for designing new treatments against cancer. However, the lack of in vivo models to study their activity has curbed their consideration as anticancer molecules," explains JeanPhilippe Parvy, first author on the paper. Mutants for AMPs were previously not available because the large number and small size of their encoding genes made them difficult to mutate with traditional genetic tools. Here, Parvy and colleagues used a CRISPR/Cas9 strategy to generate flies with null mutant alleles for the defensin gene. To investigate the effects of Defensin on tumors, the def mutants were combined with mutation in the tumor suppressor gene discs-large (dlg).

The investigators quantified tumor volume and tumor cell death in the different fly lines. Their results show that tumor size and tumor cell death were, respectively, increased and decreased in dlg; def double mutant flies compared with $d l g$ flies, suggesting that Defensin controls tumor size via regulation of apoptosis. Parvy and colleagues also identified the main sources of Defensin in $d l g$ flies: the fat body and the trachea. Using tissue-specific gene knockdown strategies, they showed that the two tissues had nonredundant roles in Defensin-mediated tumor cell death and that the main pathways mediating innate immunity in flies, Toll and lmd, were involved in Defensin production.

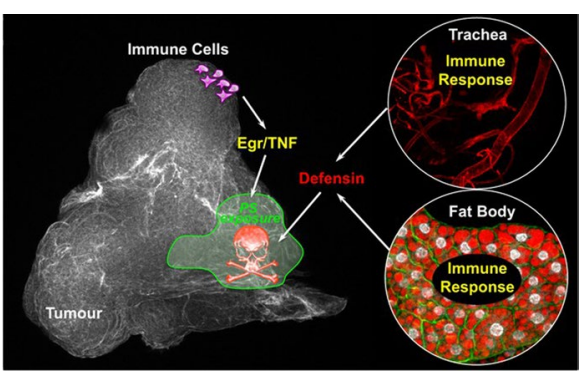

A model for the antitumoral activity of Defensin. Reprinted from Parvy et al. (2019) eLife Sciences Publications.

Def overexpression in the fat body of dlg;def double mutants partially restored cell death and reduced tumor burden. Likewise, injection of synthetic Defensin increased tumor cell death in the imaginal discs of both $\mathrm{dlg}$ and $\mathrm{dlg}$; def larvae but had no effect on tissues from wild-type larvae. As AMPs are positively charged molecules that recognize negatively charged molecules exposed on the cell surface of pathogens during infection, Parvy et al hypothesized that the AMP selectively kills tumor cells while sparing normal cells by using a similar mechanism. They showed that a negatively charged phospholipid becomes present at the surface of cells undergoing tumorigenesis and that this modification in the cell membrane lipid composition is selectively recognized by Defensin.

The study also provides insights on the molecular mechanisms driving Defensinmediated tumor cell death, notably the role of TNF. "Previous work has shown that the killing activity of TNF - a central molecule in cancer immunity conserved from flies to human - required activation of the immune response in non-tumor tissue. We hypothesized that AMPs could be the immune-induced signal mediating tumor cell death by TNF," explains Parvy.

The team had previously shown that the release of TNF homolog Eiger (Egr) from macrophage-like hemocytes promotes tumor cell death in tumor-bearing flies. In this new study, they showed that egr knockdown in hemocytes decreased tumor- cell surface-exposed phosphatidylserine; injection of Defensin promoted tumor cell death in $d l g$ mutants but not in Egrdeficient flies. Altogether, these results demonstrate that the cellular immune response to tumors in Drosophila involves the binding of hemocytes to tumor cells and the activation of the TNF pathway, which induces phosphatidylserine exposure. This first response is followed by a humoral response in the fat body and trachea that triggers cancer cell death via the action of Defensin.

Parvy says that their work raises several interesting questions: how does the AMP kills tumor cells? How does TNF induce changes in lipid composition of the tumor cell membrane? Is this antitumoral effect restricted to Defensin, or can other fly AMPs also kill tumor cells? Do AMPs work together to promote tumor killing? How do tumors induce a strong and systemic immune response? Does Defensin kill other tumor cells or is this effect restricted to some specific tumor cells? The team will try to address some of these questions in their future work by using adult Drosophila tumor models, notably fly models of colorectal cancer.

Human Defensins are not homologous to Drosophila AMPs but the molecules of the two species show structural resemblances. Human B-Defensin-1 (hBD1) has been shown to be downregulated in prostate cancer and renal clear cell carcinomas, and to induce cell death in prostate and renal cancer cells in vitro. Further studies are required to determine if the anticancer activity displayed by Defensins from different species is linked to their structural properties.

"We believe that our in vivo model represents a great opportunity to understand in details the key molecular mechanisms allowing AMPs to kill cancer cells and this is a key step toward their potential use in anticancer therapy," concludes Parvy.

\section{Alexandra Le Bras}

Published online: 16 September 2019 https://doi.org/10.1038/s41684-019-0404-9 